\title{
BLOCK-WISE 3D ULTRASOUND IMAGE SUPER-RESOLUTION
}

\author{
Nwigbo Kenule Tuador ${ }^{1}$, Pham Duong Hung ${ }^{1}$, Varray François ${ }^{2}$, Basarab Adrian $^{1}$, Kouame Denis ${ }^{1}$ \\ ${ }^{1}$ University of Toulouse, Université Paul Sabatier, IRIT, CNRS UMR 5505 \\ ${ }^{2}$ University of Lyon, INSA-Lyon, UCBL, CNRS, Inserm, CREATIS UMR 5220, U1206, F-69621 Lyon, France
}

\begin{abstract}
This paper addresses the problem of 3D ultrasound (US) single image super-resolution (SR), i.e., recover a high-resolution volume from its blurred, decimated, and noisy version. A new 3D US SR technique based on a linear forward model is studied by taking into account the axial variability of the point spread function (PSF) within a block-wise recovery process. The PSF is estimated using a recent algorithm along the axial direction with the assumption that it is isotropic in the other two spatial directions. By exploiting the linear image formation model, a cost function is constructed from a data fidelity term penalized by an $l_{1}$-norm regularization function imposing the sparsity of the solution. Numerical results show the efficiency of the proposed method when compared to the observed image.
\end{abstract}

Index Terms - 3D ultrasound image super-resolution, homomorphic filtering, ADMM.

\section{INTRODUCTION}

3D single image super-resolution (SR) has attained increasing interest from the medical imaging community in the last few years. It can indeed be encountered in a wide range of applications e.g., brain magnetic resonance imaging analysis [1], cardiac applications [2] or dental cone beam computed tomography [3]. The main goal of 3D-SR methods is to recover a 3D high-resolution (HR) image from its acquired low-resolution (LR) counterpart, enabling a better image visualization and content analysis [4]. Many related works have been proposed in the literature, among which learning-based approaches and those based on linear image formation models are the most common. The former typically rely on convolutional neural networks whose parameters are obtained by a training process that makes a connection between LR images and corresponding HR images [1, 2, 3]. Unfortunately, these learning-based approaches have a very high computational complexity while the training process generally requires a large dataset which is not always available in many practical situations. Instead, the latter formulate the SR image as an inverse image restoration problem. It consists in minimizing a cost function including regularizations such as total variation (TV) [5], low-rank [6] or wavelet domain-based sparsity [7]. In spite of their interest, these methods have many significant drawbacks such as an unsuitability to spatial variability of the point spread function (PSF), a high computational burden, and the fact that $3 \mathrm{D}$ volumes are unfolded into either $2 \mathrm{D}$ matrices or $1 \mathrm{D}$ vectors resulting in information loss on adjacent voxels.

To mitigate these limitations, inspired by our recent modelbased work for 3D SR problem developed in [8], we introduce, in this paper, a novel fast 3D ultrasound (US) SR method based on a non-unfolding technique and a block-wise approach, leading to an HR block-wise (BW) super-resolved image. More precisely, the non-unfolding technique consists in handling simultaneously the associated decimation and blurring operators. It is based on studying their intrinsic properties in the frequency domain that enables a computational cost reduction, while the BW approach takes into account the variation of the PSF in the axial dimension. This proposed method is denoted by 3D BW-SR in the sequel.

The remaining part of this paper is structured as follows. In Section 2 we introduce a US image formation model and its associated 3D SR technique. Then, Section 3 outlines the 3D BW-SR method. Section 4 presents the numerical results on experimental data. Finally, conclusions and future works are given in Section 5

\section{BACKGROUND}

\subsection{Ultrasound image degradation model}

This paper investigates an SR problem applied to 3D US imaging. Assuming linear US wave propagation, under the first Born approximation, post-beamformed radiofrequency (RF) images are expressed as a convolution between a spatially variant PSF and the tissue reflectivity function (TRF) to be recovered [9, 10,11]. In order to deal with the low-resolution nature of the acquired images, we introduce a decimation operator $\mathbf{D}$. In the following, we simplify this model by assuming an invariant PSF within an image block, which locally leads to the US image formation model [8]:

$$
\mathbf{y}=\mathbf{D H x}+\mathbf{n},
$$

where $\mathbf{y} \in \mathbb{R}^{N_{l}}\left(N_{l}=m_{l} \times n_{l} \times s_{l}\right)$ is an observed block extracted from the $3 \mathrm{D}$ RF volume, $\mathbf{x} \in \mathbb{R}^{N_{h}}\left(N_{h}=m_{h} \times n_{h} \times s_{h}\right)$ is the TRF to be estimated and $\mathbf{n}$ is an additive white Gaussian noise with variance $\sigma^{2}$, all arranged in lexicographical order with $N_{h}$ and $N_{l}$ being the number of image samples in $\mathbf{x}$ and $\mathbf{y}$ respectively. $\mathbf{H}$ is assumed to be block circulant with circulant blocks which accounts for the 3D convolution with the PSF and together with its conjugate transpose $\mathbf{H}^{H}$ can be decomposed as [12]:

$$
\mathbf{H}=\mathbf{F}^{H} \boldsymbol{\Lambda} \mathbf{F} \quad \text { and } \quad \mathbf{H}^{H}=\mathbf{F}^{H} \boldsymbol{\Lambda}^{H} \mathbf{F} .
$$

In (1), $\mathbf{H x}$ is decimated by the operator $\mathbf{D}$ with an integer rate of $d=d r \times d c \times d s$, i.e., $N_{h}=N_{l} \times d$. The pixel resolution loss in each spatial direction between the observed RF volume and the TRF are $d_{r}, d_{c}$ and $d_{s}$ which satisfies $m_{h}=m_{l} \times d_{r}, n_{h}=n_{l} \times d_{c}$ and $s_{h}=s_{l} \times d_{s}$.

\subsection{Super-resolution problem formation}

The main purpose of this work is to estimate the TRF from RF image as established in the model (1). From a Bayesian viewpoint, one can estimate the TRF by using the standard maximum a posteriori (MAP) estimator by maximizing the posterior distribution

$$
p(\mathbf{x} \mid \mathbf{y}) \propto p(\mathbf{y} \mid \mathbf{x}) p(\mathbf{x}),
$$

where $p(\mathbf{x})$ is the prior probability density function of $\mathbf{x}$ and the like- 


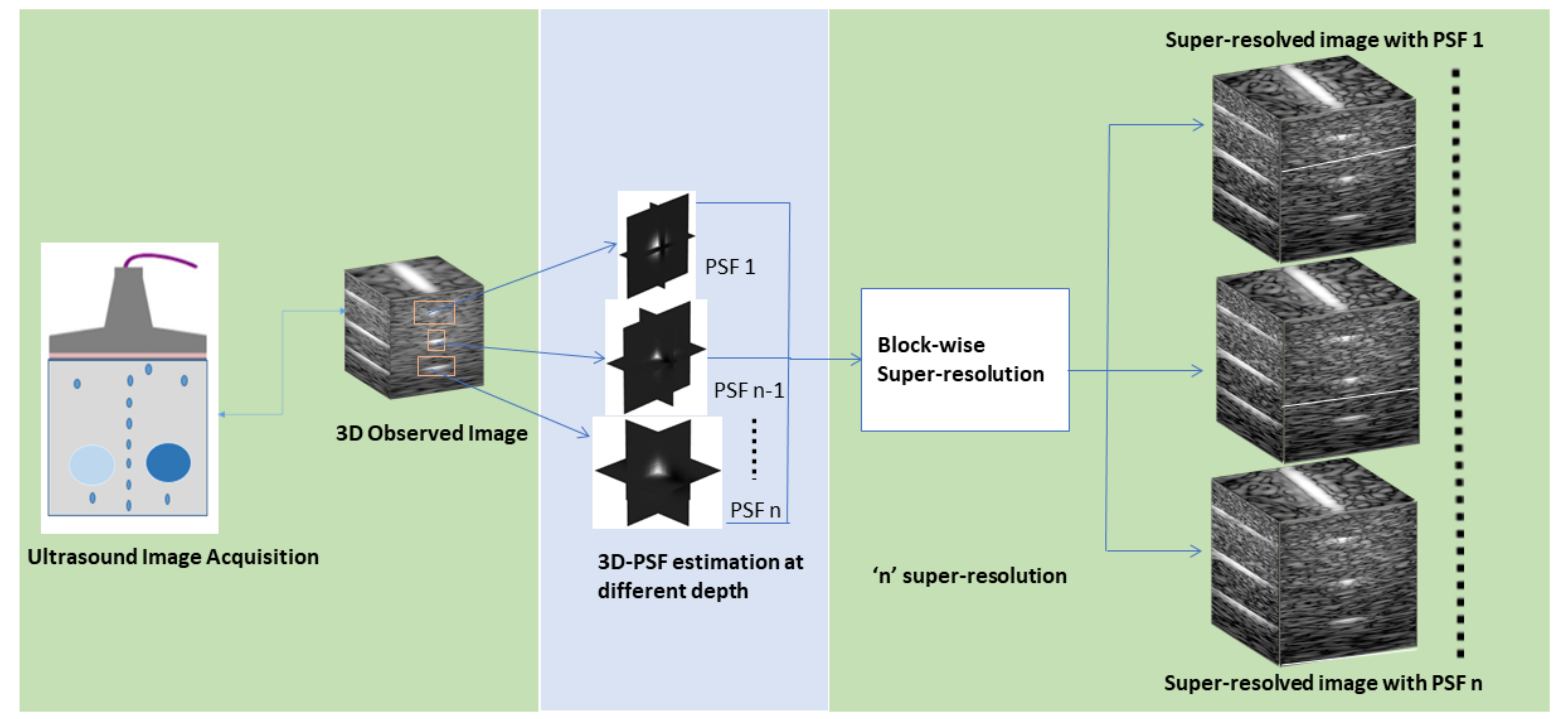

Fig. 1. Example of PSF estimation and image super-resolution using three estimated PSFs.

lihood function $p(\mathbf{y} \mid \mathbf{x})$ is given by:

$$
p(\mathbf{y} \mid \mathbf{x}) \sim \mathcal{N}\left(\mathbf{D H} \mathbf{x}, \sigma^{2} \mathbf{I}_{N_{l}}\right),
$$

where $\mathcal{N}$ stands for the Gaussian distribution and $\mathbf{I}_{N_{l}}$ is the identity matrix of size $N_{l} \times N_{l}$. The negative $\log$-posterior of $\mathbf{x}$ is given by:

$$
-\log p(\mathbf{x} \mid \mathbf{y}) \propto \underbrace{\frac{1}{2}\|\mathbf{y}-\mathbf{D H x}\|_{2}^{2}}_{\text {data fidelity term }}+\underbrace{\log (p(\mathbf{x}))}_{\text {regularization term }} .
$$

Considering a sparse assumption on the TRF, which is in consistency with a Laplace prior resulting into an $l_{1}$ regularization term, $\mathbf{x}$ can be estimated by solving the following optimization problem:

$$
\hat{\mathbf{x}}=\underset{\mathbf{x}}{\operatorname{argmin}} \frac{1}{2}\|\mathbf{y}-\mathbf{D H x}\|_{2}^{2}+\lambda\|\mathbf{x}\|_{1},
$$

where $\lambda>0$ is a hyper-parameter that weights the compromise between data fidelity term and the sparse regularization term. In what follows, we present an efficient algorithm to solve (6), whose numerical implementation is referred to as a 3D-fast SR (3D-FSR) algorithm and denoted by 3D-FSR- $l_{2}-l_{1}$.

\section{PROPOSED 3D BLOCK-WISE SUPER-RESOLUTION}

In this section, we formally present the technique used in handling the SR problem presented in Section 2.2. The method used in this study accounts for a stationary PSF in each image block. Firstly, the beamformed 3D RF image is split into $n$ overlapping axial blocks, having $n$ 3D PSFs to be estimated at $n$ different depths from the RF image (see Fig. 1). Secondly, we obtain the solution of (6) (3D-FSR$l_{2}-l_{1}$ ) for each block. Finally, we present the proposed 3D BW-SR algorithm after which the super-resolved images from each block are merged to obtain the desired TRF as highlighted in Fig. 2

\subsection{D PSF estimation}

$\mathbf{H}$ operator is formed based on a 3D PSF estimated as follows. First, a 2D PSF is estimated from the central 2D slice extracted from the current block, using the method in [13, 14]. Second, this resulting
2D PSF is extended to a 3D PSF based on the assumption that it is isotropic in the azimuthal and lateral directions, which is a reasonable hypothesis for images acquired with a 2D matrix probe.

\subsection{D Fast $\mathrm{SR}$ with $l_{1}$-norm regularization}

To efficiently solve (6), we propose to use an algorithm based on an alternating direction method of multipliers (ADMM) scheme [15]:

$$
\begin{aligned}
(\hat{\mathbf{x}}, \hat{\mathbf{u}})=\underset{\mathbf{x}, \mathbf{u}}{\operatorname{argmin}} & \frac{1}{2}\|\mathbf{y}-\mathbf{D H x}\|_{2}^{2}+\lambda\|\mathbf{u}\|_{1} \\
\text { subject to } & \mathbf{x}=\mathbf{u} .
\end{aligned}
$$

The associated augmented Lagrangian function is

$$
\mathcal{L}(\mathbf{x}, \mathbf{u}, \mathbf{d})=\frac{1}{2}\|\mathbf{y}-\mathbf{D H} \mathbf{x}\|_{2}^{2}+\lambda\|\mathbf{u}\|_{1}+\frac{\mu}{2}\|\mathbf{x}-(\mathbf{u}-\mathbf{d})\|_{2}^{2} .
$$

For the linear constraints, $\mu$ is the penalty parameter and $\mathbf{d}$ is the Lagrangian multiplier. ADMM minimizes 8 by iteratively solving subproblems over the variable $\mathbf{x}$ using our recent approach for fast 3D SR based on handling concurrently the decimation and blurring operators in the Fourier domain [8], then $\mathbf{u}$ using vector soft thresholding and updating the scaled dual variable d:

$$
\begin{aligned}
& \text { For } i=0, \ldots \\
& \qquad \begin{array}{l}
\mathbf{x}^{i+1}=\underset{x}{\operatorname{argmin}} \frac{1}{2}\|\mathbf{y}-\mathbf{D H} \mathbf{x}\|_{2}^{2}+\frac{\mu}{2}\left\|\mathbf{x}-\left(\mathbf{u}^{i}-\mathbf{d}^{i}\right)\right\|_{2}^{2} \\
\mathbf{u}^{i+1}=\underset{u}{\operatorname{argmin}} \lambda\|\mathbf{u}\|_{1}+\frac{\mu}{2}\left\|\mathbf{x}^{i+1}-\left(\mathbf{u}-\mathbf{d}^{i}\right)\right\|_{2}^{2} \\
\mathbf{d}^{i+1}=\mathbf{d}^{i}+\left(\mathbf{x}^{i+1}-\mathbf{u}^{i+1}\right)
\end{array}
\end{aligned}
$$

\subsection{Proposed 3D BW-SR algorithm}

The resulting algorithm able to solve efficiently the SR problem for each image block in the RF image is given in Algorithm 1 .

As a result, $n$ RF restored images are estimated using the SR algorithm above applied to blocks extracted from the RF volume, with the $n$ estimated PSFs (see Fig. 1). Ideally, these restored images should be merged to produce the final restored TRF but this might incur some sewing effect. In order to avoid this issue, we defined for each PSF a corresponding weighting function $w_{j}$ which reaches its maximum at the depth where the $j$ th PSF is estimated [17]. As 


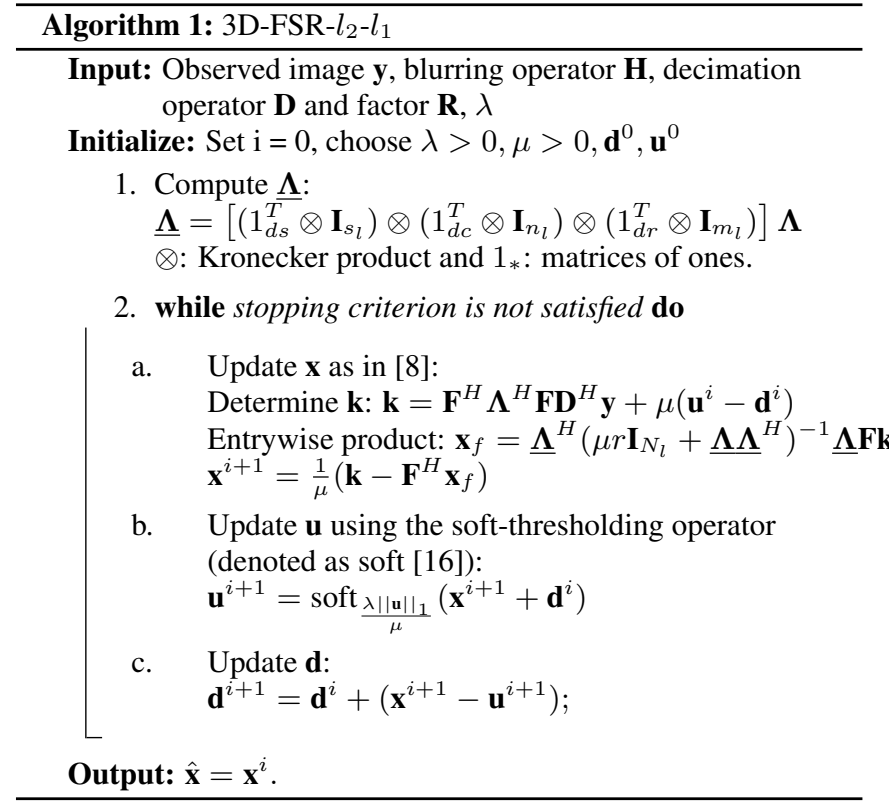

illustrated in Fig. 2 continuous windows along the $z$-axis are used to define the amplitudes of the different weights which are normalised. Finally, the restored TRF $T$ is obtained by merging the $n$ restored images following linear combinations:

$$
T=\sum_{j}^{n} w_{j} \hat{\mathbf{x}}_{j},
$$

where $\hat{\mathbf{x}}_{j}$ is the result in the region of the $j$ th PSF [17].

\section{NUMERICAL RESULTS}

The performance of the proposed method is evaluated on a real dataset, acquired using 4 synchronized Verasonics Vantage systems and a 3D 1024 elements probe [18]. 81 titled plane waves were transmitted on a commercial phantom containing three wires with a $3 \mathrm{MHz}$ sinusoidal excitation. The proposed method was tested on the post-beamforming resulting RF volume as described hereafter.

In this experiment, the RF image was divided into three blocks for both PSF estimation and block-wise image recovery process. The solution of the proposed 3D BW-SR method was compared to the observed image. As a metric of spatial resolution, the full width at half maximum (FWHM) computed around the wire locations was calculated. The results of this experiment are all shown in Fig. 3 Visually judging these plots, the SR result in Fig. 3 (b) obtained by 3D BW-SR shows a superior improved spatial resolution over the observed image in Fig. 3 (a). Quantitatively, we computed FWHM values at different depths of the three wires in both the observed image and the 3D BW-SR solution, respectively, and their gains (gains $_{\mathrm{FWHM}}=\mathrm{FWHM}_{\mathrm{observed}} / \mathrm{FWHM}_{3 \mathrm{D} \mathrm{BW}-\mathrm{SR}}$ ). The quantitative results are reported in Fig. 3 (c). They demonstrate the advantage of the proposed algorithm, which is consistent with the above qualitative observation about the SR performance of the studied techniques.

\section{CONCLUSIONS}

The main goal of this paper was to study the role of a non-stationary PSF in the restoration of TRF from the RF image. The problem was formulated as an SR problem in which a PSF estimation step was implemented in a preprocessing step. In this work, allowing the spatial variability of the PSF to be considered, an efficient blockwise restoration approach was presented. The 3D BW-SR herein does not present sewing artifacts, and is easy to implement. Further work can be devoted to jointly estimate the PSF and the TRF as a generalization of the method and to the case of non-isotropic PSF.

\section{ACKNOWLEDGMENTS}

This work has been supported by the PTDF overseas scholarship under the government of Nigeria. It was also performed within the framework of the LABEX PRIMES (ANR-11-LABX-0063) of the Universite de Lyon, within the programme 'Investissements d'Avenir' (ANR-11-IDEX-0007), operated by the French National Research Agency (ANR). This material is based upon work done on the PILoT facility (PILoT, INSA-Lyon).

\section{REFERENCES}

[1] C.-H. Pham et al., "Multiscale brain MRI super-resolution using deep 3D convolutional networks," Comput. Med. Imaging Graph, vol. 77, p. 101647, Oct. 2019.

[2] O. Oktay et al., "Multi-input cardiac image super-resolution using convolutional neural networks," in Lect. Notes Comput. Sci. Springer International Publishing, 2016, pp. 246-254.

[3] J. Hatvani et al., "Deep learning-based super-resolution applied to dental computed tomography," IEEE Trans. Radiat. Plasma Med. Sci., vol. 3, no. 2, pp. 120-128, 2019.

[4] S. C. Park, M. K. Park, and M. G. Kang, "Super-resolution image reconstruction: a technical overview," IEEE Signal Process. Mag., vol. 20, no. 3, pp. 21-36, 2003.

[5] A. Toma et al., "Total variation super-resolution for 3D trabecular bone micro-structure segmentation," in 22nd Proc. in EUSIPCO, 2014, pp. 2220-2224.

[6] F. Shi et al., "Lrtv: Mr image super-resolution with low-rank and total variation regularizations," IEEE Trans. Med. Imaging, vol. 34, no. 12, pp. 2459-2466, 2015.

[7] $\mathrm{X}$. Zhang and $\mathrm{X}$. Wu, "Image interpolation by adaptive 2-D autoregressive modeling and soft-decision estimation," IEEE Trans. Image Process., vol. 17, no. 6, pp. 887-896, 2008.

[8] N. K. Tuador, D. H. Pham, J. Michetti, A. Basarab, and D. Kouamé, "A novel fast 3D single image super-resolution algorithm," in 18th Proc. IEEE Int. Symp. Biomed. Imaging (ISBI), 2021, pp. 73-76.

[9] J. Ng et al., "Modeling ultrasound imaging as a linear, shiftvariant system," IEEE Trans. Ultrason Ferroelectr. Freq. Control, vol. 53, no. 3, pp. 549-563, 2006.

[10] J. Jensen, "Field: A program for simulating ultrasound systems," Med. Biol. Eng. Comput., vol. 34, pp. 351-352, 1996.

[11] J. Jensen and N. Svendsen, "Calculation of pressure fields from arbitrarily shaped, apodized, and excited ultrasound transducers," IEEE Trans. Ultrason Ferroelectr. Freq. Control, vol. 39, no. 2, pp. 262-267, 1992.

[12] P. C. Hansen, J. G. Nagy, and D. P. O. Leary, Deblurring Images. SIAM J. Appl. Math, Jan. 2006.

[13] O. Michailovich, A. Basarab, and D. Kouame, "Iterative reconstruction of medical ultrasound images using spectrally constrained phase updates," in 16th Proc IEEE Int. Symp. Biomed. Imaging. (ISBI), 2019, pp. 1765-1768. 


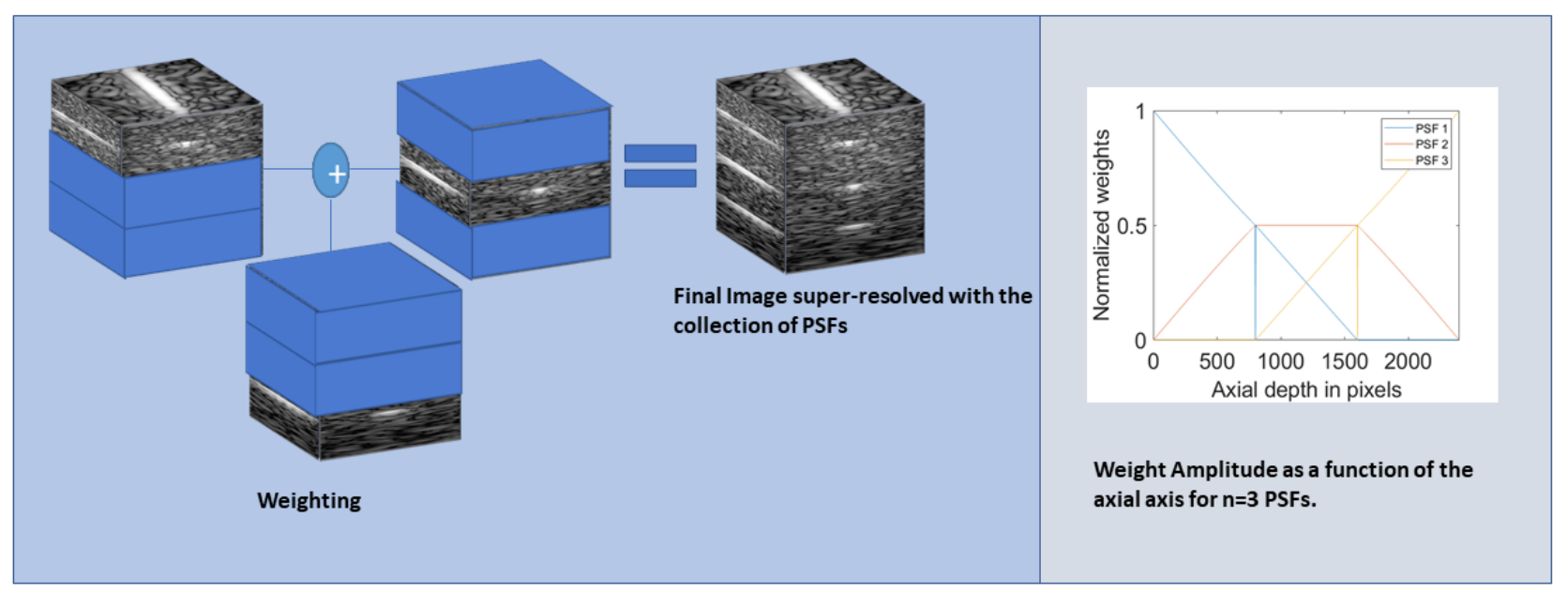

Fig. 2. The block weight principle and the weight functions $w_{j}$ corresponding to various PSFs as a function of depth.

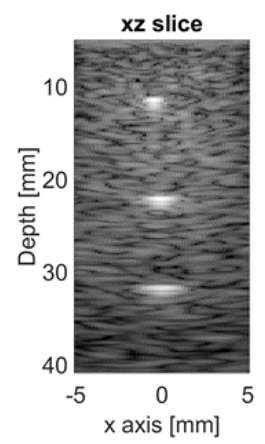

(a)

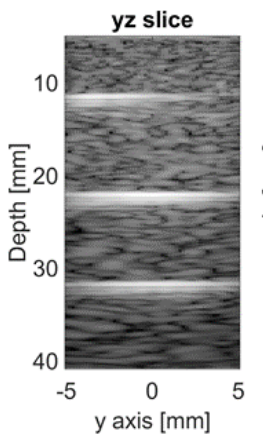

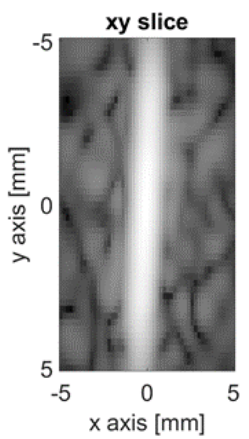

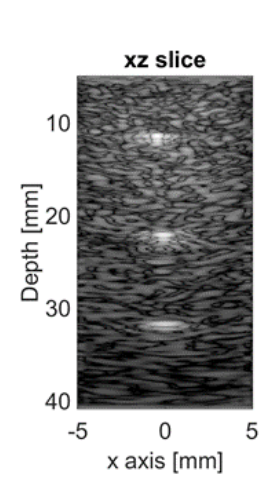

(b)

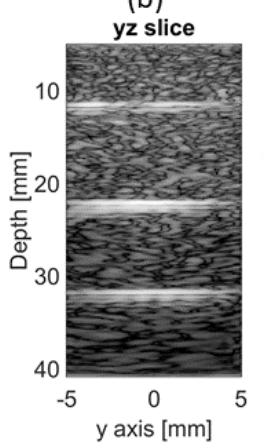

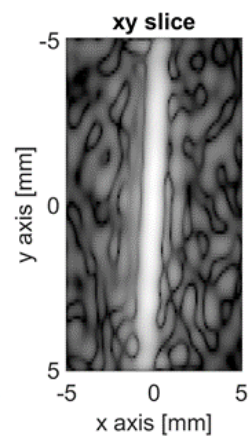

(c)

\begin{tabular}{|c|c|c|c|}
\hline \multicolumn{3}{|c|}{ FWHM measures } \\
\hline Positions in depth & FWHM $_{\text {observed }}$ & FWHM $_{3 \mathrm{D} \text { BW-SR }}$ & $\begin{array}{c}\text { gains }_{\mathrm{FWHM}}=\mathrm{FWHM}_{\text {observed }} / \\
\text { FWHM }_{3 \mathrm{D} \text { BW-SR }}\end{array}$ \\
\hline $\mathrm{z}=11.54 \mathrm{~mm}$ & $1.04 \mathrm{~mm}$ & $0.32 \mathrm{~mm}$ & 3.25 \\
\hline $\mathrm{z}=22.17 \mathrm{~mm}$ & $1.56 \mathrm{~mm}$ & $0.58 \mathrm{~mm}$ & 2.69 \\
\hline $\mathrm{z}=31.85 \mathrm{~mm}$ & $2.08 \mathrm{~mm}$ & $0.88 \mathrm{~mm}$ & 2.36 \\
\hline
\end{tabular}

Fig. 3. Real data experiment: (a) observed image, (b) SR result using the proposed 3D BW-SR algorithm, (c) the FWHM measures for each wire at each depth in both observed image and the 3D BW-SR solution, and their gains. Note that these are slices from the central axial direction of each resolution.

[14] D.-H. Pham, A. Basarab, I. Zemmoura, J.-P. Remenieras, and D. Kouamé, "Joint blind deconvolution and robust principal component analysis for blood flow estimation in medical ultrasound imaging," IEEE Trans. Ultrason Ferroelectr. Freq. Control, vol. 68, no. 4, pp. 969-978, 2021.

[15] S. Boyd et al., "Distributed optimization and statistical learning via the alternating direction method of multipliers," Found. Trends Mach. Learn., vol. 3, no. 1, pp. 1-122, 2010.

[16] Z. Chen, A. Basarab, and D. Kouamé, "Reconstruction of enhanced ultrasound images from compressed measurements us- ing simultaneous direction method of multipliers," IEEE Trans. Ultrason Ferroelectr. Freq. Control, vol. 63, 072016.

[17] F. Varray, O. Bernard, S. Assou, C. Cachard, and D. Vray, "Hybrid strategy to simulate 3-D nonlinear radio-frequency ultrasound using a variant spatial psf," IEEE Trans. Ultrason Ferroelectr. Freq. Control, vol. 63, no. 9, pp. 1390-1398, 2016.

[18] L. Petrusca, F. Varray, R. Souchon, A. Bernard, J.-Y. Chapelon, H. Liebgott, W. A. N'Djin, and M. Viallon, "Fast volumetric ultrasound b-mode and doppler imaging with a new high-channels density platform for advanced $4 \mathrm{~d}$ cardiac imaging/therapy," Applied Sciences, vol. 8, no. 2, 2018. 